\title{
GPS SCHEDULING WITH GRACEFUL RATE ADAPTATION *
}

\author{
Anastasios Stamoulis \\ Department of Electrical Engineering \\ University of Virginia \\ Charlottesville, VA 22903
}

\author{
Jörg Liebeherr \\ Polytechnic University \\ Department of Electrical Engineering \\ Brooklyn, NY 11201
}

\begin{abstract}
This paper addresses a shortcoming of the widely used Generalized Processor Sharing (GPS) scheduling algorithm which can have significant impact on the provided service, however, which has been given little attention. Since, with GPS, the service rate received by a session is proportional to the number of backlogged sessions in the system, the service rate of a session may change abruptly if some other session becomes active. This may result in abrupt increases of delay of consecutive packets. In this paper, we present and analyze a new scheduler, called Slow-Start GPS ( $\left.S^{2} G P S\right)$, which alleviates the problem. $S^{2} G P S$ is a modification of GPS where a session does not receive its guaranteed service rate immediately after it becomes active. Instead, the service rate of a session is gradually increased.
\end{abstract}

\section{Introduction}

The Generalized Processor Sharing (GPS) scheduling method is known to support isolation and sharing in a QoS network $[2,4,5]$. In recent years many researchers have studied GPS scheduling in the context of packet switching $[1,2,3,6,7,9,10,11]$. GPS can provide rate guarantees to the sessions it services. However, with GPS, a session that has been active for a long period of time can experience dramatic decreases in its service rate when some other previously idle session becomes active. The decrease of the service rates can be quite large, resulting in a possibly significant increase of the delay of consecutive packets of an active session.

In this study we show how to alleviate the problem of abrupt decrease of service rates with GPS. We propose a modification to GPS, called Slow-Start GPS ( $S^{2}$ GPS), that prevents abrupt rate changes and delay increases by gracefully degrading the service rate of active sessions. This is accomplished by the following modification to the original GPS scheduling method. Whenever a session becomes active and starts sending packets, this session is not assigned the full bandwidth at once, but gradually. The name "slow-start" was elected to indicate that the service rate of a newly active session is slowly increased when the session starts transmitting. As a result, the service rates of previously active sessions decrease smoothly.

The remainder of this paper is structured as follows. In Section 2 we discuss GPS and its packetized version, PGPS. In Section 3 we study a class of scheduling disciplines that alleviate the problem of abrupt degradation of service and we present the novel $\mathrm{S}^{2}$ GPS scheduler. In Section 4 we analyze the worst-case delays with $S^{2}$ GPS. In Section 5 we define the packetized version of $S^{2}$ GPS and show how it can be implemented using the concept of virtual time. Since space limitations do not allow us to include examples in this paper, we refer to [8] for a set of

\footnotetext{
* This work is supported in part by the National Science Foundation under Grant No. NCR-9309224 and NCR-9624106.
}

simulation experiments.

\section{GPS/PGPS Scheduler}

\subsection{GPS Server}

A Generalized Processor Sharing (GPS) scheduler is a work-conserving ${ }^{1}$ scheduler that serves the incoming traffic at a fixed rate $r$. Each session $i$ that is served by the scheduler is characterized by a weight $\phi_{i}$. Let $S_{i}(\tau, t)$ be the amount of session $i$ traffic that is served in the interval $(\tau, t]$. Define a session as backlogged whenever the queue $Q_{i}$ of this session is not zero. Then, a GPS scheduler is defined as one for which

$$
S_{i}(\tau, t) / S_{j}(\tau, t) \geq \phi_{i} / \phi_{j}
$$

for any pair of sessions $i$ and $j$. If $B(t)$ is the set of backlogged sessions at time instant $t$, then every session $i$ in $B(t)$ is served at the instantaneous service rate of:

$$
r_{i}(t)=r \phi_{i} / \sum_{j \in B(t)} \phi_{j}
$$

Therefore, a session $i$ is guaranteed a minimum service rate of $g_{i}$ for any time interval that it is backlogged:

$$
g_{i}=r \phi_{i} / \sum_{j=1}^{N} \phi_{j}
$$

where $N$ is the maximum number of sessions that are being served by the GPS scheduler.

GPS is an idealized scheduler in that it assumes that traffic is infinitely divisible; hence, it can serve all backlogged sessions simultaneously. However, in reality, only one session can receive service at a time, and a packet has to be fully transmitted before another packet starts being served. Thus, in actual networks the operations of GPS must be approximated. The most popular approximation of GPS is Packet-By-Packet Generalized Processor Sharing (PGPS) [4] which is defined as follows. Let $d_{i, g p s}^{(p)}$ be the departure time of a packet $p$ from session $i$ under GPS. Then, PGPS is the service discipline that transmits packets in increasing order of $d_{g p s}^{(p)}$ 's.

In [4], it is proved that:

$$
d_{i, P G P S}^{(k)}-d_{i, G P S}^{(k)} \leq L_{\max } / r \quad \forall i, k
$$

where $d_{i, P G P S}^{(k)}, d_{i, G P S}^{(k)}$ are the departure times of the $k$ th packet of session $i$ under GPS. In other words, a PGPS system cannot fall behind a GPS system by more than one maximum packet size. In [4], it was proved that for leaky bucket constrained sessions, GPS guarantees deterministic worst-case delays.

\footnotetext{
${ }^{1} \mathrm{~A}$ scheduler is work-conserving if it is not idle if there is incoming
} traffic to be transmitted. Otherwise, it is non-work-conserving. 


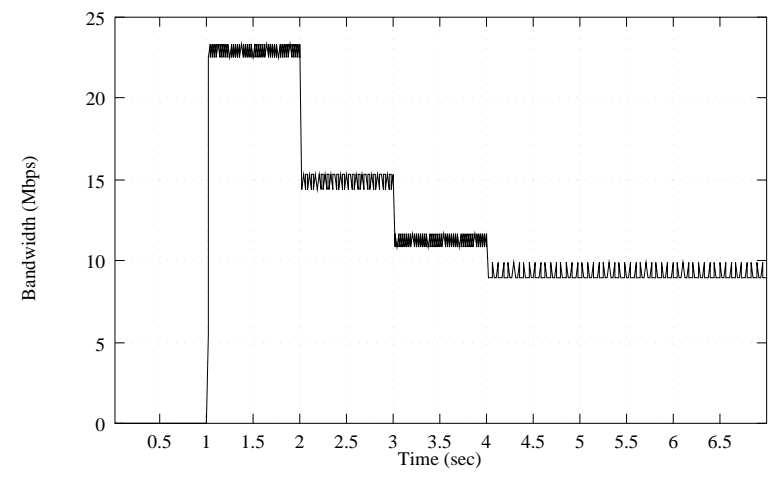

Figure 1: Available bandwidth for Session 1 under GPS

\subsection{GPS and abrupt decrease of service rates}

When new sessions start transmitting packets, the service rates of the previously active sessions decrease abruptly. This abrupt decrease, which in some cases can be dramatic, can result in abrupt increases of delay and jitter. The decrease of service rates is a direct result of the fact that, under GPS, the service rate that a session receives is dependent on the number of backlogged sessions (as (2) indicates).

Let us present an example that illustrates how the service rate of a session under GPS can decrease rapidly. Suppose that we have a switch that operates at 45 Mbps. Further suppose that the switch serves five sessions. All sessions have the same weights, i.e., $\phi_{0}=\phi_{1}=\phi_{2}=\phi_{3}=$ $\phi_{4}$. The guaranteed rate for every session is $9 \mathrm{Mbps}$. Let us assume that session 0 becomes active at time $t=0 \mathrm{sec}$, session 1 at $t=1 \mathrm{sec}, \ldots$, session 4 at time $4 \mathrm{sec}$.

In Figure 1 we plot the bandwidth that is available to session 1 in such a scenario. ${ }^{2}$ As the figure indicates, when session 1 starts transmitting at time $t=1 \mathrm{sec}$, it immediately obtains its fair share of the bandwidth, which is 22.5 Mbps. When sessions 2, 3, and 4 start transmitting, the available bandwidth for session 1 is decreased rapidly to $15 \mathrm{Mbps}, 11.25 \mathrm{Mbps}$ and $9 \mathrm{Mbps}$, respectively. The figure clearly shows that GPS abruptly changes the service rates of a session whenever a new session becomes active. ${ }^{3}$

\section{Slow-Start GPS Schedulers $\left(S^{2} G P S\right)$}

In GPS, changes in the service rates occur when the set of the backlogged sessions changes. This happens when either "new" sessions become active or some sessions cease to be backlogged. When "new" sessions become backlogged, they demand their share of the bandwidth. This results in the decrease of the service rates of the "old" sessions and a potential increase of delays. On the other hand, when some sessions are no longer backlogged, the service rates of all other sessions have to be increased abruptly. In this paper we are only interested in the first case. Handling of the second case can be done by making the scheduler non-work-conserving.

Our approach is based on the following basic idea: when a new session $k$ with $\phi_{k}$ becomes active at time instant $t_{k}$ (We use $t_{k}$ to denote the time instant when session $k$ becomes active after an idle period), then the weight of this session will be gradually increased from an initial value of $\phi=0$ to its final value, $\phi_{k}$. Since the new session will not

${ }^{2}$ The figure depicts the results for PGPS, a packetized version of GPS (see Section 6). Note that the fluctuations of the service rate are caused by the fact that PGPS is an approximation of GPS. As a result, the total available bandwidth oscillates.

${ }^{3}$ In Figure 3 we show how our slow-start mechanism for GPS smoothes the changes of the service rate. receive at once its fair, we anticipate that the service rates of all other sessions will not drop dramatically.

In a slow-start GPS scheduler, for each new session $k$, we use $T_{k}>0$ to specify the length of the slow-start period, that is, the amount of time that has to pass before session $k$ is assigned its fair share of the bandwidth. If session $k$ becomes active at $t_{k}$, its service rate is increased in the interval $\left[t_{k}, t_{k}+T_{k}\right]$, and at time $t_{k}+T_{k}$, the session has obtained its fair share of the bandwidth. Let us denote the instantaneous service rates as $\hat{r}_{k}(t)$. A slow-start GPS scheduler is a work-conserving scheduler that maintains two sets of sessions, $B(t)$ and $B_{\text {new }}(t) . B(t)$ is the set of active sessions at time $t$, and $B_{\text {new }}(t)=\left\{k \mid \hat{r}_{k}(t)<\right.$ $\left.r_{k}(t)\right\}$ is the set of all newly active sessions that have not yet acquired their fair share of the bandwidth at time $t$.

The slow-start GPS scheduler is characterized by the following properties:

1. The service rate of a newly active session in the slowstart phase is an increasing function in time. Thus, $0 \leq$ $\hat{r}_{k}(t) \leq \hat{r}_{k}(t+\Delta t) \leq r_{i}\left(t+T_{k}\right)$ for $t_{k} \leq t<t+\Delta t \leq$ $t_{k}+\overline{T_{k}}$.

2. If session $k$ is backlogged throughout the interval $\left[t_{k}, t_{k}+T_{k}\right]$, then after time $t_{k}+T_{k}$, session $k$ is served at a rate at least as large as the service rate under GPS Note that $\hat{r}_{k}(t)$ can be greater than $r_{k}(t)$ for $t \geq t_{k}+T_{k}$. This will happen when some session (other than $k$ ) is in the slow-start phase. Thus, $\hat{r}_{k}(t) \geq r_{k}(t)$ for $t \geq t_{k}+T_{k}$. 3. For any two connections $i$ and $j$ in $B(t)-B_{\text {new }}(t)$, we have that $\hat{r}_{i}(t) / \hat{r}_{j}(t)=\phi_{i} / \phi_{j}, \forall t$.

In this paper we investigate a slow-start GPS scheduler where the increase of service rates is carried out linearly with respect to time. Also, we assume that the length of the slow start period is identical for all sessions, that is, $T_{k}=$ $T, \forall k$. Then the service rate of a session $k \in B_{\text {new }}(t)$ that becomes active at time $t_{k}$ and is continuously backlogged in the interval $\left[t_{k}, t_{k}+T\right]$ is given by:

$$
\hat{r}_{k}(t)=\frac{t-t_{k}}{T} r(t) \quad: t_{k} \leq t \leq T+t_{k}
$$

At time $t=T+t_{k}, k$ is removed from $B_{\text {new }}(t)$ because it will have been assigned its fair share of the bandwidth. We refer to this scheduler as Slow-Start GPS ( $\mathbf{S}^{2}$ GPS).

In Figure 2 we illustrate the difference between GPS and $\mathrm{S}^{2} \mathrm{GPS}$. This figure depicts the service rate of session $k$ as a function of time. The figure shows three events: At time $t_{k}$, session $k$ becomes active, at time $t_{j}$, session $j$ becomes active and at time $t_{x}$ session $k$ becomes idle. Under GPS, the service rate function $r_{k}(t)$ for a session $k$ consists of linear horizontal segments. Under $S^{2}$ GPS the service rate function does not change abruptly at the points in time where a session becomes active or a session leaves the system.

If $B(t)$ and $B_{\text {new }}(t)$ are constant in $\left[t_{k}, T+t_{k}\right]$, then a session $k \in B_{\text {new }}(t)$ is served at the instantaneous rate of:

$$
\hat{r}_{k}(t)=\frac{t-t_{k}}{T} \frac{\phi_{k}}{\sum_{i \in B(t)} \phi_{i}} r \quad: t_{k} \leq t \leq T+t_{k}
$$

and a session $j \in B(t)-B_{\text {new }}(t)$ is served at the instantaneous rate of:

$$
\hat{r}_{j}(t)=\frac{\phi_{j}\left(r-\sum_{k \in B_{\text {new }}(t)} \hat{r}_{k}(t)\right)}{\sum_{i \in B(t)-B_{\text {new }}(t)} \phi_{i}} \quad: t_{k} \leq t \leq T+t_{k}
$$




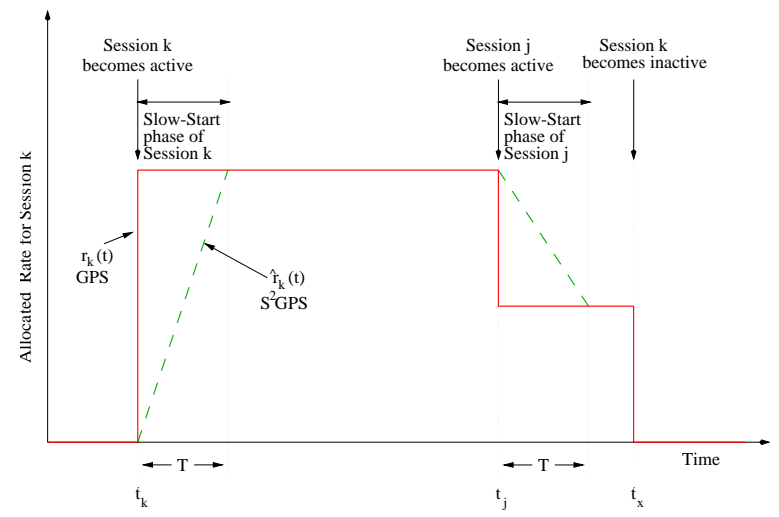

Figure 2: Service rate of Session $k$ as a function of time in GPS and $\mathrm{S}^{2}$ GPS.

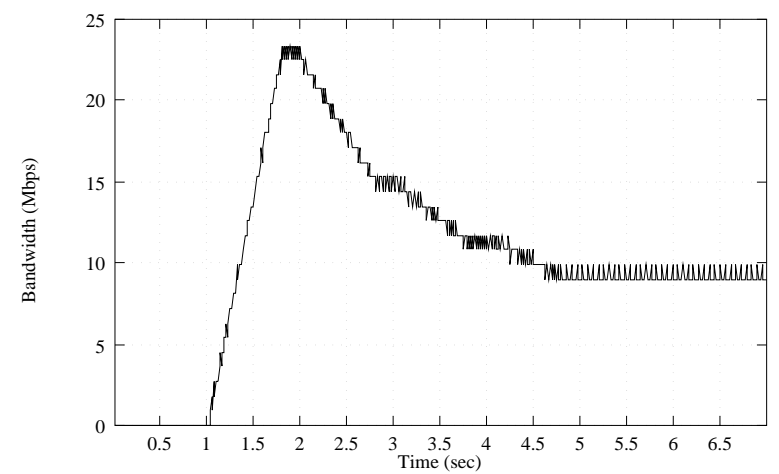

Figure 3: Available bandwidth for Session 1 under $S^{2}$ GPS with $T=0.8 \mathrm{sec}$.

Equations (6) and (7) illustrate the fact that when a session is in the slow-start phase, the bandwidth, which corresponds to the difference between the fair share and the actual service rate, is distributed among the "old" sessions.

In Figure 3 we present the available bandwidth of a session for a ${ }^{2}$ GPS scheduler with $T=0.8 \mathrm{sec}$. The input parameters are identical to those used for Figure 1.

One can make the following observations about $S^{2}$ GPS First, equation (1) still holds for any two sessions that belong to $B(t)-B_{\text {new }}(t)$. Second, in the long term, when all sessions are active and their service rates have assumed their steady-state values, a $S^{2}$ GPS system behaves exactly in the same way as a GPS system. Third, if the duration $T$ of the slow-start phase is small relatively to the time that a session is active, then $S^{2}$ GPS will not necessarily cause a dramatic increase to the average delay of the session. However, depending on the value of $T$, we expect the worst-case delay in a slow-start GPS scheduler to be larger than that in GPS.

\section{Analysis of $\mathbf{S}^{2}$ GPS}

The linear change of service rate in $\mathrm{S}^{2}$ GPS increases the worst-case delay of new sessions that arrive in the system. In the following we derive bounds for the worst-case delay.

In $S^{2}$ GPS, when a previously idle session becomes active, it is assigned its fair share of the bandwidth only gradually. As a result, we expect the worst-case delay in a $S^{2}$ GPS system to be larger than the worst-case delay in the corresponding GPS system. For sessions that have been assigned their fair share of the scheduler bandwidth, the worst-case delay in $S^{2}$ GPS system is expected to be the same as in a GPS system, as the session will receive service at a minimum rate of $g_{i}$. Therefore, the difference between the worst-case delays in $\mathrm{S}^{2}$ GPS and GPS system for a session $i$ is obtained by evaluating the difference of service that the session receives until it is assigned its fair share of the bandwidth. In the following we will derive the worst-case delay bound for sessions that are constrained by leaky buckets.

In our analysis we take advantage of the so-called isolation property of GPS (which is also retained in $\mathrm{S}^{2} \mathrm{GPS}$ ). For the calculation of the worst-case delay, we consider a $\mathrm{S}^{2}$ GPS system where a maximum of $N$ sessions can be admitted. Without loss of generality, we will calculate the worst-case delay for the $k$-th session that starts transmitting at time 0 . As in GPS, in $\mathrm{S}^{2}$ GPS, session $k$ experiences its worst-case delay when: (1) all the other sessions in the system are continuously backlogged, and (2) session $k$ is greedy, i.e., $A_{k}(0, t)=\sigma_{k}+\rho_{k} t$. The first condition keeps the maximum service rate attained by session $k$ to the minimum rate $g_{k}$. The second condition is required because the worst-case scenario will occur when a session transmits at its maximum allowable rate.

Let $Q_{k}(t)$ be the queue size (or backlog) of session $k$ at time instant $t$. Then we have:

$$
Q_{k}(t)=A_{k}(0, t)-S_{k}(0, t)
$$

Let $\delta_{k}(t)$ be the delay of each arrival at time $t$. Then we have:

$$
Q_{k}(t)=S_{k}\left(t, t+\delta_{k}(t)\right)
$$

For ease of notation, we will use $A_{k}(t)$ for $A_{k}(0, t)$ and $S_{k}(t)$ for $S_{k}(0, t)$. Figure 4 depicts $A_{k}(t)$ and $S_{k}(t)$. As Figure 4 suggests, $\delta_{k}(t)$ is the horizontal distance between $A_{k}(t)$ and $S_{k}(t) ; Q_{k}(t)$ is the vertical distance between $A_{k}(t)$ and $S_{k}(t)$.

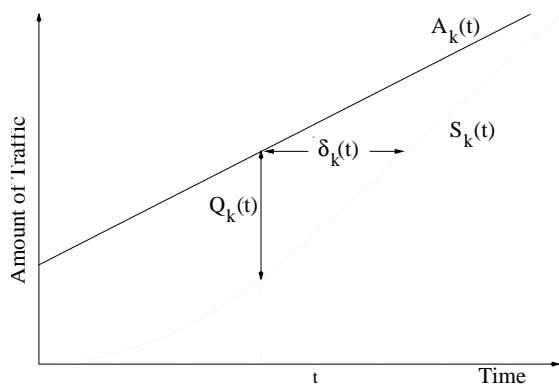

Figure 4: Relation of $\delta_{k}(t), Q_{k}(t), A_{k}(t)$, and $S_{k}(t)$

Under $\mathrm{S}^{2}$ GPS, the service rate of session $k$ is given by:

$$
r_{k}(t)=\left\{\begin{aligned}
\frac{t}{T} g_{k} & : \quad t \leq T \\
g_{k} & : \quad t>T
\end{aligned}\right.
$$

The amount of traffic $S_{k}\left(t_{1}, t_{2}\right)$ that is served in the interval $\left[t_{1}, t_{2}\right]$ and the instantaneous service rate are associated through the equation:

$$
S_{k}\left(t_{1}, t_{2}\right)=\int_{t_{1}}^{t_{2}} r_{k}(t) d t
$$

From (9) and (11) we have:

$$
Q_{k}(t)=\int_{t}^{t+\delta_{k}(t)} r_{k}(\tau) d \tau
$$


From (8) and (12) we obtain:

$$
\begin{gathered}
S_{k}(0, t)+\int_{t}^{t+\delta_{k}(t)} r_{k}(\tau) d \tau=A_{k}(0, t) \\
\text { As } S_{k}(0, t)=\int_{0}^{t} r_{k}(\tau) d \tau: \\
\sigma_{k}+\rho_{k} t=\int_{0}^{t+\delta_{k}(t)} r_{k}(\tau) d \tau
\end{gathered}
$$

Equation (13) will be used to evaluate the delay $\delta(t)$ as a function of time $t$. Hence, we are able to calculate the maximum delay $\delta_{\max }$, which is given in the following theorem. (Refer to [8] for a complete proof of Theorem 1.)

Theorem 1 The worst-case delay $\delta_{k}^{\max }$ of the $\left(\sigma_{k}, \rho_{k}\right)$ constrained session $k$ is:

$$
\delta_{k}^{\text {max }}=\left\{\begin{array}{llr}
\frac{T}{2}+\frac{\sigma_{k}}{g_{k}} & : & T<\frac{2 \sigma_{k}}{g_{k}} \\
\sqrt{\frac{2 T \sigma_{k}}{g_{k}}} & : & \frac{2 \sigma_{k}}{g_{k}} \leq T<\frac{2 \sigma_{k} g_{k}}{\rho_{k}^{2}} \\
\frac{\sigma_{k}}{\rho_{k}}+\frac{\rho_{k} T}{2 g_{k}} & : & \frac{2 \sigma_{k} g_{k}}{\rho_{k}^{2}} \leq T
\end{array}\right.
$$

provided that $g_{k} \geq \rho_{k}$.

\section{A Packet-by-packet version of $S^{2}$ GPS}

$S^{2}$ GPS assumes a fluid model where traffic is infinitely divisible. In reality, however, a scheduler can serve one packet at a time. In this section we define the packet approximation of $S^{2}$ GPS, called Slow-Start Packetized Generalized Processor Sharing or $S^{2}$ PGPS. Also, we show how $S^{2}$ GPS can be implemented using the concept of virtual time.

\section{1 $\mathrm{S}^{2}$ PGPS}

In this Subsection, we define the packet approximation of $S^{2}$ GPS, called $S^{2}$ PGPS. S ${ }^{2}$ PGPS is the scheduling discipline that transmits packets in increasing order of their finishing times under the $\mathrm{S}^{2}$ GPS system. $\mathrm{S}^{2}$ GPS attempts to approximate the fluid model as closely as possible. Note that $S^{2}$ PGPS is derived from $S^{2}$ GPS in the same way that PGPS is derived from GPS [4]. The question that arises is whether $S^{2}$ PGPS is a good approximation of a $S^{2}$ GPS system. We will prove that this is indeed the case. Specifically, we can show that a $S^{2}$ PGPS system cannot fall behind from the corresponding $S^{2}$ GPS system by more than one maximum packet size. We will take advantage of the following results that are available for GPS/PGPS [4]:

1. Let $p$ and $q$ be packets in a GPS system at time $\tau$, and suppose that packet $p$ completes service before packet $q$ if there are no arrivals after time $\tau$. Then, the packet $p$ will also complete service before the packet $q$ for any pattern of arrivals after time $\tau$.

2. Let $\hat{F}_{p}, F_{p}$ be the times that packet $p$ departs from the PGPS and GPS systems, respectively. Then for all packets $p, \hat{F}_{p}-F_{p} \leq \frac{L_{\max }}{r}$.

3. Let $\hat{S}_{i}(0, \tau)$ be the amount of service that session $i$ receives under $S^{2}$ GPS. Then, for all times $\tau$ and for each session $i S_{i}(0, \tau)-\hat{S}_{i}(0, \tau) \leq L_{\max }$.

It is not hard to show that the above properties also apply in $S^{2}$ GPS. This is because the proofs of these properties for PGPS, which are given in [4], are not sensitive to time-dependent service rates. Thus, a $S^{2}$ PGPS system cannot fall behind from the corresponding $S^{2}$ GPS system by more than one packet size. These properties facilitate the translation of delay bounds under a $S^{2}$ GPS system to the corresponding S $^{2}$ PGPS system.

\subsection{Virtual Time Implementation of $\mathbf{S}^{2}$ PGPS}

In this section we present an implementation of the $S^{2}$ PGPS based on virtual times. The concept of virtual time as a means of implementing PGPS was proposed in $[2,4]$; the virtual time $V(t)$ in $[2,4]$ is used as a measure of progress in the system. When packets arrive, the scheduler assigns virtual time deadlines to them and serves packets in increasing order of these deadlines. The virtual time is set to zero at the beginning of a busy period and increases at the marginal rate of $1 / \sum_{i \in B(t)} \phi_{i}$. Thus, during any system busy period $\left[t_{1}, t_{2}\right], V(t)$ evolves as follows:

$$
\begin{aligned}
V\left(t_{1}\right) & =0 \\
\partial V(t) / \partial t & =1 / \sum_{i \in B(t)} \phi_{i} \quad: t_{1} \leq t \leq t_{2}
\end{aligned}
$$

which yields:

$$
V(t)=\int_{t_{1}}^{t_{2}} 1 / \sum_{i \in B(t)} \phi_{i} d \tau
$$

Let us define as an event in the system the arrival or the departure of a packet. Let $e_{j}$ be the time that the $j$-th event in the system occurs. By observing that $B(t)$ is constant in any time interval during which no events occur, we obtain:

$$
V\left(e_{j}+\tau\right)=V\left(e_{j}\right)+\tau / \sum_{i \in B\left(e_{j}\right)} \phi_{i} \quad 0 \leq \tau \leq e_{j+1}-e_{j}
$$

For the $p$-th packet of the $k$-th session, the virtual start time $S_{i}^{(p)}$ and virtual finish time $F_{i}^{(p)}$ are defined as:

$$
\begin{gathered}
S_{i}^{(p)}=\max \left\{F_{i}^{(p-1)}, V\left(t_{i}^{(p)}\right)\right\} \\
F_{i}^{(p)}=S_{i}^{(p)}+L_{i}^{(p)} / \phi_{i}
\end{gathered}
$$

where $t_{i}^{(p)}$ is the arrival time of packet $p$ and $L_{i}^{(p)}$ is the length of packet $p$. The scheduler serves packets in increasing order of their virtual finishing times.

An implementation of $S^{2}$ PGPS with virtual time is not straightforward and must address the following two problems:

1. From (18) we have that the virtual time $V(t)$ is calculated as a function of the $\phi$ 's of the sessions. Note, however, that the weights of the sessions in the slowstart phase have to be modified to reflect the increasing service rates that these sessions receive, and the decreasing service rates of the other, i.e., the previously active sessions.

2. For a packet $p$ that is transmitted during the slowstart phase of a $S^{2} G P S$ of a session $k$, the service rates of the session at the beginning and at the end of the transmission will be different. Correspondingly, the weight $\phi_{k}$ of a session $k$ will take different values during the transmission of a packet. As (20) suggests, the deadline of a packet depends on the $\phi_{k}$ of the session. If the $\phi_{k}$ of session $k$ is not constant over the transmission of a packet of the session, then it is not obvious how a deadline can be assigned to this packet.

We proceed to present solutions to these two problems. In Subsection 5.3 we show how the weights of sessions in the slow-start phase can be calculated. In Subsection 5.4 we show how the virtual finishing times are calculated in $S^{2}$ GPS 


\subsection{Definition of $\phi_{k}(t)$}

Our goal is to define the $\phi_{k}$ of every session $k$ in $B_{\text {new }}(t)$ as a function of time, $\phi_{k}(t)$, such that:

$$
r_{k}(t)=\frac{\phi_{k}(t)}{\sum_{j \in B(t)-B_{n e w}(t)} \phi_{j}+\sum_{j \in B_{\text {new }}(t)} \phi_{j}(t)} r
$$

Using (6), (7), and (21), we can calculate $\phi_{k}(t)$ for every session in the slow-start phase. We show how this can be done in the case when (i) only one session $k$ is in the slowstart phase, and (ii) the set $B(t)$ of the backlogged sessions is constant in the time interval $\left[t_{k}, t_{k}+T\right]$. In Subsection 5.5 we discuss how this restriction can be relaxed.

To derive $\phi_{k}(t)$, recall that the service rate of session $k$ in time interval $\left[t_{k}, t_{k}+T\right]$ is given by:

$$
r_{k}(t)=\frac{t-t_{k}}{T} \frac{\phi_{k}}{\sum_{i \in B(t)} \phi_{i}} r
$$

As only session $k$ is in the slow-start phase, (21) becomes:

$$
r_{k}(t)=\frac{\phi_{k}(t)}{\sum_{i \in B(t), i \neq k} \phi_{i}+\phi_{k}(t)} r
$$

and (22) becomes:

$$
r_{k}(t)=\frac{t-t_{k}}{T} \frac{\phi_{k}}{\sum_{i \in B(t), i \neq k} \phi_{i}+\phi_{k}} r
$$

From (23) and (24) we have:

$$
\frac{\phi_{k}(t)}{\sum_{i \in B(t), i \neq k} \phi_{i}+\phi_{k}(t)}=\frac{t-t_{k}}{T} \frac{\phi_{k}}{\sum_{i \in B(t), i \neq k} \phi_{i}+\phi_{k}}
$$

which implies:

$$
\phi_{k}(t)=\frac{\frac{t-t_{k}}{T} \frac{\phi_{k}}{\sum_{i \in B(t)} \phi_{i}} \sum_{i \in B(t), i \neq k} \phi_{i}}{1-\frac{t-t_{k}}{T} \frac{\phi_{k}}{\sum_{i \in B(t)} \phi_{i}}}
$$

Equation (25) clearly shows that $\phi_{k}(t)$ is an increasing function of time $t$. At time $t=t_{k}+T$, we have that $\phi_{k}\left(t_{k}+T\right)=\phi_{k}$ which implies that session $k$ will be assigned its fair share of the bandwidth.

\subsection{Virtual Finishing Time in $\mathbf{S}^{2}$ PGPS}

Let $p$ be a packet of session $k$ that is transmitted during the slow-start phase of session $k$. In this subsection we will show how the virtual finishing time of this packet can be computed upon the arrival of the packet. Note that the virtual finishing time is used as the deadline with which the packet $p$ will be tagged upon its arrival.

As (25) suggests, the weight $\phi_{k}$ of a session $k$ in the slow-start phase changes during the transmission of a packet $p$ of this session. We calculate an average value of the weight $\phi_{k}$ of the session over the transmission of the packet and we call it the "effective" value $\phi_{e f f}^{(p)}$. Using $\phi_{e f f}^{(p)}$, it is possible to calculate the virtual finishing time as $S_{k}^{(p)}+\frac{L_{k}^{(p)}}{\phi_{e f f}^{(p)}}$. In other words, we are able to use (20) provided that we have calculated $\phi_{\text {eff }}^{(p)}$. We will show how $\phi_{\text {eff }}^{(p)}$ can be calculated for a session $k$ in the slow-start phase. We assume that only session $k$ is in the slow-start phase and that $B(t)$ is constant in $\left[t_{k}, t_{k}+T\right]$.

Let $w_{p}$ denote the elapsed time between the end of the transmission of packet $p$ and the arrival of the first packet of session $k$. Clearly, the transmission of packet $p$ ends at time $t_{k}+w_{p}$. We can calculate $w_{p}$ in terms of $w_{p-1}$. As the transmission of packet $p$ will start at $t_{k}+w_{p-1}$ and end at $t_{k}+w_{p}$, we have:

$$
\begin{aligned}
L_{k}^{(p)} & =\int_{t_{k}+w_{p-1}}^{t_{k}+w_{p}} r_{k}(t) d t \\
& =\int_{t_{k}+w_{p-1}}^{t_{k}+w_{p}} \frac{t-t_{k}}{T} \frac{\phi_{k}}{\sum_{i=1}^{N} \phi_{i}} r d t \\
& =\int_{w_{p-1}}^{w_{p}} \frac{\tau}{T} \frac{\phi_{k}}{\sum_{i=1}^{N} \phi_{i}} r d t
\end{aligned}
$$

which yields:

$$
w_{p}=\sqrt{\frac{2 L_{k}^{(p)} \sum_{i=1}^{N} \phi_{i}}{r \phi_{k}}+\left(w_{p-1}\right)^{2}}
$$

However, packet $p$ is served at a rate $\frac{r \phi_{e f f}^{(p)}}{\sum_{i=1, i \neq k}^{N} \phi_{i}+\phi_{e f f}^{(p)}}$. As the transmission of the packet takes time $w_{p}-w_{p-1}$, we have:

$$
\frac{r \phi_{e f f}^{(p)}}{\sum_{i=1}^{N} \phi_{i}+\phi_{\text {eff }}^{(p)}}\left(w_{p}-w_{p-1}\right)=L_{k}^{(p)}
$$

which yields:

$$
\phi_{e f f}^{(p)}=\frac{L_{k}^{(p)} \sum_{i=1, i \neq k}^{N} \phi_{i}}{r\left(w_{p}-w_{p-1}\right)-L_{k}^{(p)}}
$$

Using (26) and (27), we can now devise a procedure for $S^{2}$ GPS that assigns a deadline to an incoming packet of a new session. The deadlines of packets from sessions that are not in the slow-start phase can be directly calculated using (20). In the following, we present pseudo-code for the algorithm that assigns deadlines to packets from sessions that are in the slow-start phase:

procedure Assign_Deadline_To(Packet $p$, session $k$ )

1. if $p=1$

2. $\quad$ set $w_{0}=0$

3. add session $k$ to $B_{\text {new }}$

4. if $w_{p-1}<T$

5. calculate $w_{p}$ using (26)

6. calculate $\phi_{\text {eff }}^{(p)}$ using (27)

7. assign a deadline to packet $p$ using (20)

8. else $/ *$ the slow-start phase of session $k$ is over $* /$

9. remove $k$ from $B_{\text {new }}(t)$

When the first packet of session $k$ arrives, session $k$ enters a slow-start phase. Thus it is inserted to $B_{\text {new }}(t)$ and 
$w_{0}$ is initialized to 0 . Then $w_{1}$ is calculated from (26) and $\phi_{\text {eff }}^{(1)}$ is calculated from (27). A deadline to the first packet of the session packet is assigned using (20). When the $p$ th packet of session $k$ arrives, the scheduler has to check if the session is still in the slow-start phase. This check can be carried out in the following way. If the $(p-1)$-th packet departs before the end of the slow-start phase, i.e., $w_{p-1}<T$, then packet $p$ will also be transmitted during the slow-start phase of session $k$. Thus, $w_{p}$ is calculated using (26) and $\phi_{e f f}^{(p)}$ is calculated using (27). However, if $w_{p-1}>T$, then the slow-start phase of session $k$ is over. session $k$ is removed from $B_{\text {new }}(t)$ and its $\phi$ is set to $\phi_{k}$ for all the other packets from session $k$.

\subsection{Relaxing the Assumption}

So far we have shown how a $S^{2}$ PGPS system can be implemented by assigning deadlines to packets of sessions in the slow-start phase provided that the following conditions hold:

Condition $C_{1}$ : Only one session is in the slow-start phase. Condition $C_{2}: \mathrm{B}(\mathrm{t})$ is constant if a session is in the slowstart phase.

If this assumption holds, then $S^{2}$ PGPS provides the following guarantees:

Guarantee $G_{1}$ : The new session will experience a linear increase of its service rate.

Guarantee $G_{2}$ : The worst-case delay bounds as given in Equation (14) will hold.

Guarantee $G_{3}$ : Previously active sessions experience smooth decreases of their service rates.

We now relax this assumption and examine the impact on guarantees $G_{1}, G_{2}$, and $G_{3}$, if conditions $C_{1}$ or $C_{2}$ do not hold.

Let us suppose that $C_{1}$ does not hold. We will show that $G_{2}$ and $G_{3}$ still hold. When $C_{1}$ does not hold, there is a time period where $n>1$ sessions $k_{1}, k_{2}, \ldots, k_{n}$ are in the slow-start phase. Without loss of generality we can assume that session $k_{1}$ first entered the slow-start phase, then session $k_{2}$ and so on, i.e., $t_{k_{1}} \leq t_{k_{2}} \leq \ldots \leq t_{k_{n}}$. In this scenario, the increase of the service rate of session $k_{1}$ will be linear only until the time that session $k_{2}$ enters the slow-start phase. In other words, in the time interval $\left[t_{k_{1}}, t_{k_{2}}\right]$ the service rate of session $k_{1}$ increases linearly. After time $t_{k_{2}}$ session $k_{1}$ sees its service rate increase but not in a linear fashion. This is due to (27) not taking into account the $\phi$ 's of sessions $t_{k_{2}} \ldots t_{k_{n}}$. However, at time $t_{k_{1}}+T$, session $k_{1}$ is guaranteed to be served at a minimum rate $g_{k}$ as given by (3). Similarly, the service rates of sessions $k_{2}, \ldots, k_{n}$ also increase gradually but not linearly. Thus, when a new session enters the system, it is always served at a gradually increasing rate; the increase is linear only if assumptions $C_{1}$ and $C_{2}$ hold. As a result, $G_{3}$ holds.

Next we prove that $G_{2}$ holds even if $C_{1}$ does not hold. Recall that the delay bounds are computed by making worst-case assumptions, i.e., all $N-1$ sessions are continuously backlogged and only one session $k$ is in the slowstart phase. Let us denote the service rate of session $k$ as $r_{k}^{o n e}(t)$ when only session $k$ is in the slow-start phase and $r_{k}^{\text {many }}(t)$ when several sessions are in the slow-start phase. We have $r_{k}^{o n e}(t) \geq r_{k}^{\text {many }}(t)$ since $\mathrm{S}^{2}$ GPS is workconserving and a session in the slow-start phase is always served at a rate smaller than the service rate the session gets when it is not in the slow-start phase. Hence, when $C_{1}$ does not hold, a session in the slow-start does not experience the worst-case delays, and $G_{2}$ holds.

Thus, relaxing $C_{1}$ does not have any detrimental effects on $S^{2}$ PGPS. It turns out that this is also the case with re- laxing assumption $C_{2}$; we will show that $G_{2}$ and $G_{3}$ still hold. When $C_{2}$ does not hold, then some previously active sessions are removed from the system while some "new" sessions are still in the slow-start phase. If an "old" session ceases to be backlogged, its service rate is distributed to all remaining sessions in the system. The sessions in $B_{n e w}(t)$ will experience a sudden increase in the service rates. As their service rates will increase, their delays will decrease; hence, $G_{2}$ will hold. However, this increase of service rates will not be at the expense of the remaining sessions in $B(t)-B_{\text {new }}(t)$, as these sessions will also take a significant part of the bandwidth that was made available. Thus the smooth decrease of service rates is guaranteed in this case, too, and $G_{3}$ will hold. Hence, the slow-start nature of $S^{2}$ PGPS is preserved.

\section{Conclusions}

In this paper we have shown that the GPS scheduling discipline is unable to provide graceful degradation of service since the service rates of active sessions decrease abruptly when new sessions start transmitting. We have proposed and analyzed a modification to GPS, called Slow-Start GPS or $\mathrm{S}^{2}$ GPS that remedies this problem. We have shown how $S^{2}$ GPS can be implemented in packetswitched networks; the packetized version of $S^{2} \mathrm{GPS}$, $S^{2}$ PGPS, can be implemented using the concept of virtual time.

\section{References}

[1] J. C. R. Bennett and H. Zhang. WF2Q: Worst-case fair weighted fair queueing. In Proc. IEEE Infocom '96, March 1996.

[2] A. Demers, S. Keshav, and S. Shenker. Analysis and simulation of a fair queueing algorithm. In Proc. ACM Sigcomm'89, pages 1-12, 1989.

[3] S. J. Golestani. A self-clocked fair queueing scheme for broandband applications. In Proc. IEEE Infocom '94, pages 636-646, 1994.

[4] A. K. Parekh and R. G. Gallager. A generalized processor sharing approach to flow control in integrated services networks: the single-node case. IEEE/ACM Transactions on Networking, 1(3):344-357, June 1993.

[5] A. K. Parekh and R. G. Gallager. A generalized processor sharing approach to flow control in intergrated services networks: the multiple node case. IEEE/ACM Transanctions on Networking, 2:137-150, April 1994.

[6] D. Saha, S. Mukherjee, and S. H. Tripahi. Carry-over round robin: a Simple cell scheduling mechanism for ATM networks. In Proc. IEEE Infocom'96, pages 630-637, 1996.

[7] S. Shreedhar and G. Varghese. Efficient fair queueing using deficit round robin. IEEE Transanctions on Networking, 4(3):375-385, June 1996.

[8] A. Stamoulis and J. Liebeherr. $S^{2} G P S$ : Slow-Start Generalized Porcessor Sharing. Technical Report CS-97-03, University of Virginia, 1997.

[9] D. Stiliadis and A. Varma. Design and analysis of framebased fair queueing: a new traffic scheduling algorithm for packet-switched networks. In Proc. ACM Sigmetrics'96, pages 104-115, May 1996.

[10] O. Yaron and M. Sidi. Generalized processor sharing networks with exponentially bounded burstiness arrivals. Journal of High Speed Networks, 3:375-387, 1994.

[11] Z. L. Zhang, D. Towsley, and J. Kurose. Statistical analysis of generalized processor sharing scheduling discipline. In Proc. ACM Sigcomm'94, pages 68-77, August 1994. 